\title{
Unpolarized states and hidden polarization
}

\author{
P. de la Hoz, ${ }^{1}$ G. Björk, ${ }^{2}$ A. B. Klimov ${ }^{3}$ G. Leuchs, ${ }^{4,5}$ and L. L. Sánchez-Soto ${ }^{1,4,5}$ \\ ${ }^{1}$ Departamento de Óptica, Facultad de Física, Universidad Complutense, 28040 Madrid, Spain \\ ${ }^{2}$ Department of Applied Physics, Royal Institute of Technology (KTH), AlbaNova, SE-106 91 Stockholm, Sweden \\ ${ }^{3}$ Departamento de Física, Universidad de Guadalajara, 44420 Guadalajara, Jalisco, Mexico \\ ${ }^{4}$ Max-Planck-Institut für die Physik des Lichts, Günther-Scharowsky-Straße 1, Bau 24, 91058 Erlangen, Germany \\ ${ }^{5}$ Department für Physik, Universität Erlangen-Nürnberg, Staudtstraße 7, Bau 2, 91058 Erlangen, Germany
}

(Received 6 July 2014; published 15 October 2014)

\begin{abstract}
We capitalize on a multipolar expansion of the polarization density matrix, in which multipoles appear as successive moments of the Stokes variables. When all the multipoles up to a given order $K$ vanish, we can properly say that the state is $K$ th-order unpolarized, as it lacks of polarization information to that order. First-order unpolarized states coincide with the corresponding classical ones, whereas unpolarized to any order tally with the quantum notion of fully invariant states. In between these two extreme cases, there is a rich variety of situations that are explored here. The existence of hidden polarization emerges in a natural way in this context.
\end{abstract}

DOI: 10.1103/PhysRevA.90.043826

PACS number(s): 42.25.Ja, 42.50.Dv, 42.50.Ar, 42.50.Lc

\section{INTRODUCTION}

Very often an involved physical concept can be better understood in terms of its opposite. Polarization is a pertinent example: perhaps the most sensible way to look at it is to explore unpolarized states, as one can make sense of them using exclusively invariance principles, a tool of paramount importance in physics.

The constitution of unpolarized light was investigated from the very beginning of modern optics. Indeed, already Stokes [1] and Verdet [2] offered a lucid characterization of what they called "natural" light by using the projections of the intensity onto the axes of a rotated Cartesian coordinate system. Unpolarized states are those that remain invariant under any rotation of that coordinate system and under any phase shift between its rectangular components.

In classical optics, the field components of unpolarized light are well modeled by zero-mean, uncorrelated, stationary Gaussian random process [3]. The previous invariance conditions thus determine the entire probabilistic structure of the projected intensities [4]. However, as the standard theory is limited to first-order moments, unpolarized light is presented as having zero-mean Stokes vector, which in geometrical terms means that it is just the origin of the Poincaré sphere [5]. We stress, though, that this is an incomplete characterization, for it safely overlooks higher-order moments [6].

At the quantum level, the invariance requirement fixes once and for all the structure of the density matrix, as first pointed out in Refs. [7,8]: unpolarized states are maximally mixed in each subspace with a given number of photons $[9,10]$. To put it in another way, it specifies the probability distribution and, as a result, all the moments of the Stokes variables.

Nowadays, there is a widespread belief that a thorough appreciation of the subtle effects arising in the quantum world requires a careful scrutiny of higher-order polarization fluctuations. We have been advocating the use of a hierarchy of correlation functions that take into account the successive moments of the Stokes variables [11-13]. The most systematic way to accomplish this is by expanding the density matrix in multipoles [14].
The idea of unpolarized states can be directly translated in this scenario: when all the multipoles up to a given order (say $K$ ) vanish, the state lacks of polarization information up to that order and hence will be called $K$ th-order unpolarized. The classical picture matches the first-order theory, whereas the quantum condition implies that all the multipoles are identically null. Our goal here is to explore the terra incognita between these two extreme cases. In this respect, we mention that, as we shall see, this is closely related with the so-called hidden polarization, introduced by Klychko $[15,16]$.

Our paper is organized as follows: In Sec. II we concisely sketch the fundamentals needed to grasp the polarization hallmarks of quantum fields and introduce the multipoles. In Sec. III we revisit unpolarized states from the viewpoint of these multipoles, defining $K$ th unpolarized states. In Sec. IV we apply the formalism to some illuminating examples and, finally, our conclusions are briefly summarized in Sec. V.

\section{POLARIZATION STRUCTURE OF QUANTUM FIELDS}

A satisfactory description of the polarization structure of quantum fields is of utmost significance for our purposes. This is precisely the objective of this section.

\section{A. Quantum polarization sector}

Let us consider a monochromatic field specified by two operators $\hat{a}_{H}$ and $\hat{a}_{V}$, representing the complex amplitudes in two linearly polarized orthogonal modes, we indicate as horizontal $(H)$ and vertical $(V)$, respectively. The Stokes operators are [17]

$$
\begin{gathered}
\hat{S}_{x}=\frac{1}{2}\left(\hat{a}_{H}^{\dagger} \hat{a}_{V}+\hat{a}_{V}^{\dagger} \hat{a}_{H}\right), \quad \hat{S}_{y}=\frac{i}{2}\left(\hat{a}_{H} \hat{a}_{V}^{\dagger}-\hat{a}_{H}^{\dagger} \hat{a}_{V}\right), \\
\hat{S}_{z}=\frac{1}{2}\left(\hat{a}_{H}^{\dagger} \hat{a}_{H}-\hat{a}_{V}^{\dagger} \hat{a}_{V}\right),
\end{gathered}
$$

together with the total photon number

$$
\hat{N}=\hat{a}_{H}^{\dagger} \hat{a}_{H}+\hat{a}_{V}^{\dagger} \hat{a}_{V} .
$$

The superscript $\dagger$ stands for the Hermitian adjoint. In this Schwinger representation [18], these operators differ by a 
factor 1/2 from the common Stokes parameters [5], but in this way the components of the Stokes vector $\hat{\mathbf{S}}=\left(\hat{S}_{x}, \hat{S}_{y}, \hat{S}_{z}\right)$ satisfy the commutation relations of the SU(2) algebra:

$$
\left[\hat{S}_{x}, \hat{S}_{y}\right]=i \hat{S}_{z},
$$

and cyclic permutations (we use $\hbar=1$ throughout).

The noncommutability of these operators precludes the simultaneous sharp measurement of the corresponding quantities. Among other consequences, this implies that no field state (apart from the two-mode vacuum) can have definite nonfluctuating values of all the Stokes operators simultaneously. This is quantified by the uncertainty relation

$$
\Delta^{2} \hat{\mathbf{S}}=\Delta^{2} \hat{S}_{x}+\Delta^{2} \hat{S}_{y}+\Delta^{2} \hat{S}_{z} \geqslant \frac{1}{2}\langle\hat{N}\rangle,
$$

where $\Delta^{2} \hat{S}_{j}=\left\langle\hat{S}_{j}^{2}\right\rangle-\left\langle\hat{S}_{j}\right\rangle^{2}$ are the variances. In this vein, one can say that the electric vector of a monochromatic quantum field never describes a definite ellipse.

Moreover, while the Stokes operators are all Hermitian, the noncommutability makes mixed, nonsymmetric products (such as $\hat{S}_{x} \hat{S}_{y}$ ) non-Hermitian, also precluding their direct measurement.

In classical optics, the total intensity is a well-defined quantity and the Poincare sphere appears then as a smooth surface with radius equal to that intensity. In contradistinction, in quantum optics we have

$$
\hat{\mathbf{S}}^{2}=\hat{S}_{x}^{2}+\hat{S}_{y}^{2}+\hat{S}_{z}^{2}=S(S+1) \hat{\mathbb{1}},
$$

where $S=N / 2$ plays the role of the spin ( $N$ being the photon number). As fluctuations in $N$ are unavoidable (leaving aside photon-number states), we are forced to talk of a three-dimensional Poincaré space (with axis $S_{x}, S_{y}$, and $S_{z}$ ) that can be envisioned as a set of nested spheres with radii proportional to the different photon numbers that contribute significantly to the state.

We next make the important observation that

$$
[\hat{N}, \hat{\mathbf{S}}]=0 \text {. }
$$

This expresses in the quantum language the fact that polarization and intensity are separate concepts: the form of the ellipse described by the electric field (polarization) does not depend on its size (intensity).

This fact brings about remarkable simplifications. First, it means that each subspace with a fixed number of photons must be handled separately. Equivalently, in the previous onionlike picture of the Poincaré space, each shell has to be addressed independently. This can be emphasized if instead of the Fock states $\left\{\left|n_{H}, n_{V}\right\rangle\right\}$, which are an orthonormal basis of the Hilbert space of these two-mode fields, we employ the relabeling

$$
|S, m\rangle \equiv\left|n_{H}=S+m, n_{V}=S-m\right\rangle,
$$

which can be seen as the common eigenstates of $\hat{S}^{2}$ and $\hat{S}_{z}$. For each fixed $S, m$ runs from $-S$ to $S$ and these states span a $(2 S+$ 1)-dimensional invariant subspace, wherein the operators $\hat{\mathbf{S}}$ act in the standard form

$$
\begin{aligned}
\hat{S}_{ \pm}|S, m\rangle & =\sqrt{S(S+1)-m(m \pm 1)}|S, m \pm 1\rangle, \\
\hat{S}_{z}|S, m\rangle & =m|S, m\rangle .
\end{aligned}
$$

Second, from (2.6) it follows that any function of the Stokes operators $f(\hat{\mathbf{S}})$ commutes with $\hat{N}$. Therefore, the matrix elements of the density matrix $\hat{\varrho}$ (which describes the state) connecting subspaces with different values of $S$ do not contribute to $\langle f(\hat{\mathbf{S}})\rangle$. Put differently, the only accessible polarization information from any state $\hat{\varrho}$ is its block-diagonal form

$$
\hat{\varrho}_{\mathrm{pol}}=\bigoplus_{S} P_{S} \hat{\varrho}^{(S)},
$$

where $P_{S}$ is the photon-number distribution $(S=0,1 / 2,1, \ldots)$ and $P_{S} \hat{\varrho}^{(S)}$ is the reduced density matrix in the subspace with spin $S$. This $\hat{\varrho}_{\text {pol }}$ has been termed the polarization sector [19] or the polarization density matrix [20]. What matters for our purposes is that any $\hat{\varrho}$ and its associated $\hat{\varrho}_{\text {pol }}$ cannot be distinguished in polarization measurements and, accordingly, we shall be using the block-diagonal form (2.9) and drop henceforth the subscript pol.

\section{B. Polarization multipoles}

Instead of using directly the states $\{|S, m\rangle\}$, it is more convenient to expand each component $\hat{\varrho}^{(S)}$ in $(2.9)$ as

$$
\hat{\varrho}^{(S)}=\sum_{K=0}^{2 S} \sum_{q=-K}^{K} \varrho_{K q}^{(S)} \hat{T}_{K q}^{(S)}
$$

The irreducible tensor operators $T_{K q}^{(S)}$ are [21]

$$
\hat{T}_{K q}^{(S)}=\sqrt{\frac{2 K+1}{2 S+1}} \sum_{m, m^{\prime}=-S}^{S} C_{S m, K q}^{S m^{\prime}}\left|S, m^{\prime}\right\rangle\langle S, m|,
$$

with $C_{S m, K q}^{S m^{\prime}}$ being the Clebsch-Gordan coefficients that couple a spin $S$ and a spin $K(0 \leqslant K \leqslant 2 S)$ to a total spin $S$. These tensors are an orthonormal basis

$$
\operatorname{Tr}\left[\hat{T}_{K q}^{(S)} \hat{T}_{K^{\prime} q^{\prime}}^{\left(S^{\prime}\right) \dagger}\right]=\delta_{S S^{\prime}} \delta_{K K^{\prime}} \delta_{q q^{\prime}},
$$

and they have the right transformation properties: under a rotation parametrized by the Euler angles $(\alpha, \beta, \gamma)$, we have

$$
\hat{R}(\alpha, \beta, \gamma) \hat{T}_{K q}^{(S)} \hat{R}^{\dagger}(\alpha, \beta, \gamma)=\sum_{q^{\prime}} D_{q^{\prime} q}^{S}(\alpha, \beta, \gamma) \hat{T}_{K q^{\prime}}^{(S)},
$$

where the $D_{q^{\prime} q}^{S}(\alpha, \beta, \gamma)$ stands for the matrix elements of the rotation operator $\hat{R}(\alpha, \beta, \gamma)$ in the basis $|S, m\rangle$ [21].

Although at first sight they might look a bit intimidating, they are nothing but the multipoles used in atomic physics [14]. After some calculations, one can recast Eq. (2.11) as

$$
\begin{aligned}
& \hat{T}_{00}^{(S)}=\frac{1}{\sqrt{2 S+1}} \hat{\mathbb{1}}, \\
& \hat{T}_{10}^{(S)}=\sqrt{\frac{3}{(2 S+1)(S+1) S}} \hat{S}_{z}, \hat{T}_{1 \mp 1}^{(S)}=\sqrt{\frac{3}{(2 S+1)(S+1) S}} \hat{S}_{ \pm} \text {, } \\
& \hat{T}_{20}^{(S)}=\sqrt{\frac{C}{6}}\left(3 \hat{S}_{z}^{2}-\hat{S}^{2}\right), \hat{T}_{2 \mp 1}^{(S)}=\sqrt{\frac{C}{2}}\left\{\hat{S}_{z}, \hat{S}_{ \pm}\right\}, \hat{T}_{1 \mp 2}^{(S)}=\sqrt{\frac{C}{2}} \hat{S}_{ \pm}^{2},
\end{aligned}
$$

where $C=30 /[(2 S+3)(2 S+1)(2 S-1)(S+1)]$ and $\{$,$\} is$ the anticommutator. In consequence, we conclude that $\hat{T}_{K q}^{(S)}$ can be related to the $K$ th power of the Stokes operators (2.1). 
The corresponding expansion coefficients

$$
\varrho_{K q}^{(S)}=\operatorname{Tr}\left[\hat{\varrho}^{(S)} \hat{T}_{K q}^{(S) \dagger}\right]
$$

are known as state multipoles. The hermiticity imposes the symmetry condition

$$
\varrho_{K-q}^{(S)}=(-1)^{q} \varrho_{K q}^{(S)},
$$

and the positive semidefiniteness of $\hat{\varrho}^{(S)}$ forces the bound

$$
\mathcal{W}_{K}^{(S)} \equiv \sum_{q=-K}^{K}\left|\varrho_{K q}^{(S)}\right|^{2} \leqslant C_{K}^{(S)},
$$

for every $K>1$ and $C_{K}^{(S)}$ a positive constant. The quantity $\mathcal{W}_{K}^{(S)}$ is just the strength of the $K$ th multipole in the state $\hat{\varrho}^{(S)}$.

Finally, we turn to the important class of axially symmetric states [14]. They are invariant under rotations about an axis that we take as the $z$ axis. Since $D_{q q^{\prime}}^{S}(0,0, \gamma)=\exp (-i q \gamma) \delta_{q q^{\prime}}$, this implies

$$
\hat{\varrho}_{\mathrm{axsym}}^{(S)}=\sum_{K=0}^{2 S} \varrho_{K 0}^{(S)} \hat{T}_{K 0}^{(S)} .
$$

Thus, they are characterized exclusively by the multipole components $\varrho_{K 0}$. Any density operator that can be obtained from $\hat{\varrho}_{\text {axsym }}$ via an $\mathrm{SU}(2)$ transformation, represents as well an axial symmetric state, as a rotation only change the direction of the symmetry axis of the state.

Some axially symmetric systems are also invariant under the reversal of the symmetry axis (i.e., $z \rightarrow-z$ ). As this corresponds to a rotation around the $y$ axis by an angle $\pi$ and $D_{q q^{\prime}}^{S}(0, \pi, 0)=(-1)^{K+q} \delta_{q-q^{\prime}}$, we get from (2.13)

$$
\varrho_{K 0}^{(S)}=(-1)^{K} \varrho_{K 0}^{(S)},
$$

so only multipoles of even rank $K$ contribute.

\section{KTH-ORDER UNPOLARIZED STATES}

From now on, we restrict ourselves to a single component $\hat{\varrho}^{(S)}$ (i.e., a fixed number of photons). This is by no means a restriction, as the discussion can be extended in a natural way to the complete polarization density matrix in (2.9).

The full polarization information is encoded in the complete multipole distribution $\left\{\mathcal{W}_{K}^{(S)}\right\}$. However, for most of the states, only a limited number of multipoles play a substantive role and the rest of them have a small contribution. As a consequence, gaining a good feeling of the corresponding behavior may be tricky.

A way to bypass this disadvantage is to look at the cumulative distribution

$$
\mathcal{A}_{K}^{(S)}=\sum_{\ell=1}^{K} \mathcal{W}_{\ell}^{(S)} .
$$

Please, note carefully that the monopolar term has been excluded, as it is trivially isotropic for all the states. The quantity $\mathcal{A}_{K}^{(S)}$ conveys whole information up to order $K$. We know from probability that it has remarkable properties [22].

To get extra insights into this subject, let us focus, for the time being, on the key example of SU(2) coherent states (also known as spin or atomic coherent states), which can be written down as the superposition [23,24]

$$
|\theta, \phi\rangle=\sum_{m=-S}^{S} C_{S m}(\theta, \phi)|S, m\rangle,
$$

with coefficients

$$
\begin{aligned}
& C_{S m}(\theta, \phi) \\
& \quad=\left(\begin{array}{c}
2 S \\
S+m
\end{array}\right)^{1 / 2}\left(\sin \frac{\theta}{2}\right)^{S+m}\left(\cos \frac{\theta}{2}\right)^{S-m} e^{-i(S+m) \phi},
\end{aligned}
$$

and $\theta$ and $\phi$ are the polar and azimuthal angles on the sphere, respectively. If $\mathbf{n}$ is the unit vector in the direction $(\theta, \phi)$, the operator $\hat{S}_{\mathbf{n}}=\mathbf{n} \cdot \hat{\mathbf{S}}$ is the observable measured in polarization experiments [25]: coherent states can be alternatively interpreted as eigenstates of $\hat{S}_{\mathbf{n}}$

$$
\hat{S}_{\mathbf{n}}|\theta, \phi\rangle=S|\theta, \phi\rangle,
$$

from which one can confirm that they saturate the uncertainty relation (2.4) and so they are the minimum uncertainty states in polarization optics.

For these states, one can immediately find

$$
\mathcal{A}_{K, \mathrm{SU}(2)}^{(S)}=\frac{2 S}{2 S+1}-\frac{[\Gamma(2 S+1)]^{2}}{\Gamma(2 S-K) \Gamma(2 S+K+2)} .
$$

As conjectured in Ref. [13], this is the maximal value attainable for any $K$ in each subspace $S$. This nicely corroborates the amazing properties of $\mathrm{SU}(2)$ coherent states: they are maximally polarized states to any order, as one would expect from a pure state that corresponds as nearly as possible to a classical spin vector pointing in a given direction.

This maximality property suggests at once a hierarchy of degrees of polarization

$$
\mathbb{P}_{K}^{(S)}=\sqrt{\frac{\mathcal{A}_{K}^{(S)}}{\mathcal{A}_{K, \mathrm{SU}(2)}^{(S)}},}
$$

which sort the relevant polarization information up to order $K(K=1, \ldots, S)$. The experimental reconstruction reported in Ref. [13] reveals that by performing a Stokes measurement in $2 K+1$ independent directions, one can actually determine $\mathcal{A}_{K}^{(S)}$ and hence all the degrees $\mathbb{P}_{K}^{(S)}$.

We will say that a state is $K$ th-order unpolarized when $\mathbb{P}_{K}^{(S)}=0$ (which obviously implies $\mathcal{A}_{K}^{(S)}=0$; i.e., all the multipoles up to order $K$ vanish) and we will denote these states as $\hat{\varrho}_{\text {unpol, } K}^{(S)}$. Note, though, that $K$ th-order unpolarized states do carry polarization information when one looks at higher-order moments. This is referred to as hidden polarization, according to the terminology coined by Klyshko [15,16], albeit it would be better to say that such states display higher-order polarization [26].

In more physical terms, the condition of $K$ th-order unpolarization amounts to imposing that the moments $\left\langle\hat{\mathbf{S}}_{\mathbf{n}}^{\ell}\right\rangle$ are independent of the direction $\mathbf{n}$ for $\ell=1, \ldots, K$ (i.e., they are isotropic). Therefore, all the moments up to order $K$ do not show any angular structure, whereas higher-order ones do. Notice, in passing, that this is the logic beyond the recent 
proposal of anticoherent states [27]: such states point nowhere (to a given order), and this is certainly one way to serve as the opposite of a state that points, as much as possible, somewhere. From this perspective, these unpolarized states are exhibits the most nonclassical features [28,29].

For the particular case of the dipole $(K=1)$, Eq. (3.6) reduces to

$$
\mathbb{P}_{1}^{(S)}=\frac{\sqrt{\left\langle\hat{S}_{x}\right\rangle^{2}+\left\langle\hat{S}_{y}\right\rangle^{2}+\left\langle\hat{S}_{z}\right\rangle^{2}}}{S},
$$

which coincides with the standard definition [30]. First-order unpolarized states verify $\mathbb{P}_{1}^{(S)}=0$, so $\langle\hat{\mathbf{S}}\rangle=0$. This goes to the classical notion of random states, as it involves exclusively first-order moments.

When the state is unpolarized to any order, only the monopole contributes:

$$
\hat{\varrho}_{\text {unpol }}^{(S)}=\frac{1}{2 S+1} \mathbb{1}_{2 S+1},
$$

so it is a random state within each invariant subspace. This is the quantum definition, which demands that the whole probability distribution to be $S U(2)$ invariant $[7,8]$; that is,

$$
[\hat{\varrho}, \hat{\mathbf{S}}]=0 \text {, }
$$

wherefrom Eq. (3.8) follows [10]. The vacuum state is the only pure state that is unpolarized according to this definition, and unpolarized mixed states are maximally mixed in each subspace $S$. Any two-mode thermal state is hence unpolarized.

\section{MENAGERIE OF UNPOLARIZED STATES}

\section{A. Single-photon unpolarized states}

Single-photon states $(S=1 / 2)$ are fairly special: they can only be first-order unpolarized. The multipole expansion of a general single-photon state reads

$$
\hat{\varrho}^{(1 / 2)}=\varrho_{00}^{(1 / 2)} \hat{T}_{00}^{(1 / 2)}+\sum_{q} \varrho_{1 q}^{(1 / 2)} \hat{T}_{1 q}^{(1 / 2)} .
$$

Since the state has only dipolar component, quantum and classical descriptions coincide. Positivity constraints the possible values of the dipole to the range $0 \leqslant \mathcal{A}_{1}^{(1 / 2)} \leqslant 1 / 2$. The condition $\mathcal{A}_{1}^{1 / 2}=0$ fixes at once unpolarized states; viz,

$$
\varrho_{\text {unpol, } 1}^{(1 / 2)}=\frac{1}{2}\left(\begin{array}{ll}
1 & 0 \\
0 & 1
\end{array}\right) \text {. }
$$

These states are both classically and quantum unpolarized, but, like all quantum objects, can only be considered as elements of an ensemble [31].

\section{B. Two-photon unpolarized states}

For two-photon states, there are first-order (or classical) and second-order (or quantum) unpolarized states. The general condition for first-order unpolarization is

$$
\hat{\varrho}_{\text {unpol }, 1}^{(1)}=\varrho_{00}^{(1)} \hat{T}_{00}^{(1)}+\sum_{q} \varrho_{2 q}^{(1)} \hat{T}_{2 q}^{(1)},
$$

with the extra constraint of positivity.
The case of axially symmetric states deserves special attention as they can always be diagonalized via $\mathrm{SU}(2)$ rotations; viz, $\hat{\rho}_{\text {assym }}^{(1)}=\hat{R}(\alpha, \beta, \gamma) \hat{\varrho}_{d}^{(1)} \hat{R}^{\dagger}(\alpha, \beta, \gamma)$, with

$$
\begin{aligned}
\hat{\varrho}_{d}^{(1)} & =\left(\begin{array}{ccc}
\lambda_{1} & 0 & 0 \\
0 & \lambda_{2} & 0 \\
0 & 0 & \lambda_{3}
\end{array}\right) \\
& =\frac{1}{\sqrt{3}} \hat{T}_{00}^{(1)}+\frac{\lambda_{1}-\lambda_{3}}{\sqrt{2}} \hat{T}_{10}^{(1)}+\frac{1-3 \lambda_{2}}{\sqrt{6}} \hat{T}_{20}^{(1)} .
\end{aligned}
$$

The state is first-order unpolarized when $\lambda_{1}=\lambda_{3}$. Since $\operatorname{Tr}\left(\hat{\varrho}_{d}\right)=1$, we can write

$$
\hat{\varrho}_{\mathrm{unpol}, 1}^{(1)}=\left(\begin{array}{ccc}
\lambda & 0 & 0 \\
0 & 1-2 \lambda & 0 \\
0 & 0 & \lambda
\end{array}\right)
$$

and positivity enforces $0 \leqslant \lambda \leqslant 1 / 2$, i.e., $0 \leqslant \mathcal{A}_{2}^{(1)} \leqslant 2 / 3$. Both the purity $\mathcal{P}^{(1)}=\operatorname{Tr}\left\{\left[\hat{\varrho}_{d}^{(1)}\right]^{2}\right\}$ and the second-order degree $\mathbb{P}_{2}^{(1)}$ depend on $\lambda$

$$
\mathcal{P}^{(1)}=6 \lambda^{2}-4 \lambda+1, \quad \mathbb{P}_{2}^{(1)}=\sqrt{(3 \lambda-1)^{2}},
$$

while $\mathbb{P}_{1}^{(1)}=0$ as anticipated. This can be recast as

$$
\mathbb{P}_{2}^{(1)}=\sqrt{\left[3 \mathcal{P}^{(1)}-1\right] / 2} .
$$

In Fig. 1 we have plotted $\mathbb{P}_{2}^{(1)}$ as a function of the purity. The maximum degree $\mathbb{P}_{2}^{(1)}$ is attained for the pure states

$$
\left|\Psi_{\mathrm{unpol}, 1}^{(1)}\right\rangle=\frac{1}{\sqrt{2}} \sin \beta\left[e^{i \alpha}|1,1\rangle-e^{-i \alpha}|1,-1\rangle\right]+\cos \beta|1,0\rangle,
$$

and they are the transformed of the state $|1,0\rangle$ under $\mathrm{SU}(2)$ rotations $\hat{R}(\alpha, \beta, \gamma)$. Incidentally, these states have served as the thread to experimentally verify the existence of hidden polarization $[32,33]$. They coincide with the anticoherent states introduced in Ref. [27] and worked out using the Majorana representation $[34,35]$. Unfortunately, their nice geometric properties cannot be extended to mixed states (4.5).

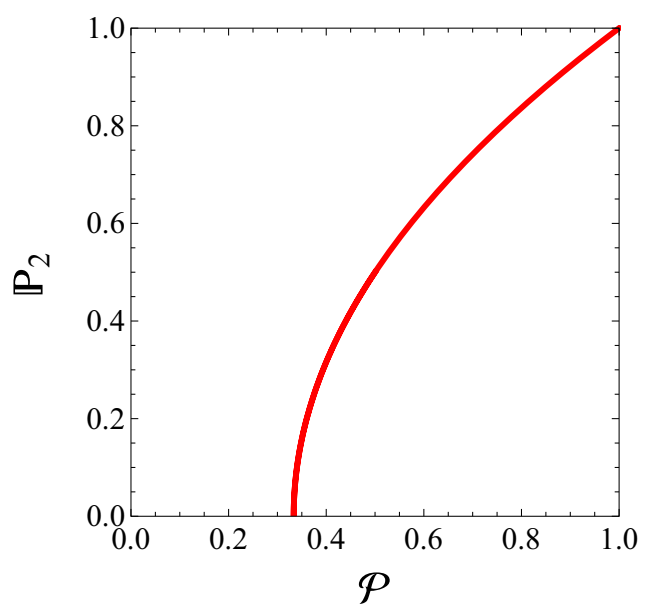

FIG. 1. (Color online) Second-order degree of polarization as a function of the purity, for the first-order unpolarized states (4.5). 


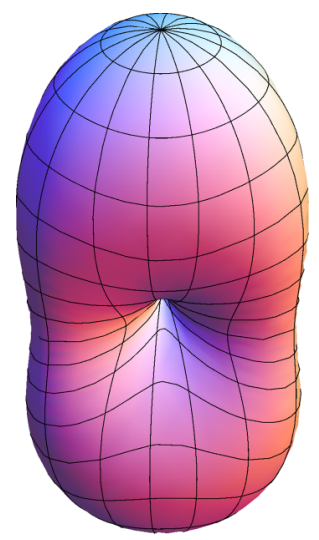

FIG. 2. (Color online) $Q$ function for three-photon first-order pure unpolarized states (4.13).

\section{Three-photon unpolarized states}

For three-photon states we have first- (classical), second-, and third-order (quantum) unpolarized states.

The diagonalizable states can be brought to the form

$$
\begin{aligned}
\hat{\varrho}_{d}^{(3 / 2)}= & \left(\begin{array}{cccc}
\lambda_{1} & 0 & 0 & 0 \\
0 & \lambda_{2} & 0 & 0 \\
0 & 0 & \lambda_{3} & 0 \\
0 & 0 & 0 & \lambda_{4}
\end{array}\right)=\frac{1}{2} \hat{T}_{00}^{(3 / 2)} \\
& -\left(\frac{2 \lambda_{2}+4 \lambda_{3}+6 \lambda_{4}-3}{2 \sqrt{5}}\right) \hat{T}_{10}^{(3 / 2)} \\
& +\left(\frac{1}{2}-\lambda_{2}-\lambda_{3}\right) \hat{T}_{20}^{(3 / 2)} \\
& +\left(\frac{-4 \lambda_{2}+2 \lambda_{3}-2 \lambda_{4}+1}{2 \sqrt{5}}\right) \hat{T}_{30}^{(3 / 2)}
\end{aligned}
$$

The condition for first-order unpolarization is

$$
2 \lambda_{2}+4 \lambda_{3}+6 \lambda_{4}-3=0,
$$

and the dipole is absent. Now there are not axially symmetric first-order unpolarized states, because when $S$ is a half integer, no state of the basis lacks first-order polarization.

The diagonal operator of a three-photon first-order unpolarized state reads

$$
\begin{aligned}
& \hat{\varrho}_{\text {unpol, } 1}^{(3 / 2)} \\
& \quad=\left(\begin{array}{cccc}
\lambda_{3}+2 \lambda_{4}-1 / 2 & 0 & 0 & 0 \\
0 & -2 \lambda_{3}-3 \lambda_{4}+3 / 2 & 0 & 0 \\
0 & 0 & \lambda_{3} & 0 \\
0 & 0 & 0 & \lambda_{4}
\end{array}\right),
\end{aligned}
$$

and positivity translates into $0 \leqslant \lambda_{3}+2 \lambda_{4}-1 / 2 \leqslant 1$ and $0 \leqslant-2 \lambda_{3}-3 \lambda_{4}+3 / 2 \leqslant 1,0 \leqslant \lambda_{3}, \lambda_{4} \leqslant 1$. The purity $\mathcal{P}$ is

$$
\mathcal{P}=\frac{1}{4}+\frac{5}{4}\left(2 \lambda_{3}+2 \lambda_{4}-1\right)^{2}+\left(\lambda_{3}+3 \lambda_{4}-1\right)^{2} .
$$

with the bounds $1 / 4 \leqslant \mathcal{P} \leqslant 5 / 8$.

We remark that by using the Majorana representation mentioned above, one can check that the SU(2)
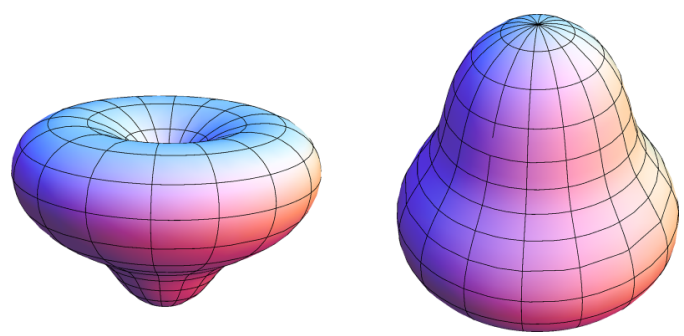

FIG. 3. (Color online) $Q$ function for axially symmetric three-photon second-order unpolarized states with maximal purity. In the left, we represent the state $3 / 4|3 / 2,1 / 2\rangle\langle 3 / 2,1 / 2|+$ $1 / 4|3 / 2,-3 / 2\rangle\langle 3 / 2,-3 / 2|$, while in the right the state $1 / 3|3 / 2,3 / 2\rangle\langle 3 / 2,3 / 2|+1 / 2| 3 / 2,-1 / 2\rangle\langle 3 / 2,-1 / 2|+1 / 6| 3 / 2$, $-3 / 2\rangle\langle 3 / 2,-3 / 2|$ is plotted.

transformed of

$$
\left|\Psi_{\text {unpol, } 1}^{(3 / 2)}\right\rangle=\frac{1}{\sqrt{2}}|3 / 2,-3 / 2\rangle+\frac{1}{\sqrt{2}}|3 / 2,3 / 2\rangle,
$$

are first-order unpolarized, although they do not fall in the class of states defined by (4.11). To better appreciate these states, one can work out the $\operatorname{SU}(2) Q$ function, which is defined as $[36,37]$

$$
Q^{(S)}(\theta, \phi)=\left\langle\theta, \phi\left|\hat{\varrho}^{(S)}\right| \theta, \phi\right\rangle,
$$

where $|\theta, \phi\rangle$ are $\operatorname{SU}(2)$ coherent states. In Fig. 2 we plot this $Q$ function for the state (4.13).

To get a second-order unpolarized state, we need the additional condition: $\lambda_{3}+3 \lambda_{4}-1=0$ and, the diagonal form for these states is

$$
\hat{\varrho}_{\text {unpol }, 2}^{(3 / 2)}=\left(\begin{array}{cccc}
\frac{1}{2}-\lambda_{4} & 0 & 0 & 0 \\
0 & 3 \lambda_{4}-\frac{1}{2} & 0 & 0 \\
0 & 0 & 1-3 \lambda_{4} & 0 \\
0 & 0 & 0 & \lambda_{4}
\end{array}\right) .
$$

The maximal purity of a second-order unpolarized axially symmetric three-photon state is $\mathcal{P}=7 / 18$. In Fig. 3, we represent the $Q$ function for second-order unpolarized states maximizing the purity.

\section{CONCLUDING REMARKS}

Multipolar expansions are a powerful machinery. We have applied such an expansion to the polarization density matrix, showing how the multipoles quantify higher-order fluctuations in the Stokes variables. In this way, we have provided a systematic characterization of unpolarized states as those states whose multipoles up to a given order vanish.

The formalism can be extended to systems in which SU(2) symmetry plays a crucial role (such as Bose-Einstein condensates, spin chains, etc.) and to other unitary symmetries, such as $\mathrm{SU}(3)$ (which is pivotal to understanding the polarization of the near field).

\section{ACKNOWLEDGMENTS}

Financial support from the Swedish Foundation for International Cooperation in Research and Higher Education 
(STINT), the Swedish Research Council (VR) through its Linnæus Center of Excellence ADOPT and Contract No. 621-2011-4575, the Mexican CONACyT (Grant No.
106525), the EU FP7 (Grant Q-ESSENCE), and the Spanish MINECO (Grant No. FIS2011-26786) is gratefully acknowledged.
[1] G. G. Stokes, Trans. Cambridge Phil. Soc. 9, 399 (1852).

[2] E. Verdet, Leçons d' Optique Physique, edited by A. Levistal, Vol. 2 (Imprimerie Impériale, Paris, 1869), pp. 78-98.

[3] N. G. van Kampen, Stochastic Processes in Physics and Chemistry, 2nd ed. (Elsevier, Amsterdam, 2007).

[4] R. Barakat, J. Opt. Soc. Am. A 6, 649 (1989).

[5] M. Born and E. Wolf, Principles of Optics, 7th ed. (Cambridge University Press, Cambridge, 1999).

[6] H. Paul and J. Wegmann, Opt. Commun. 112, 85 (1994).

[7] H. Prakash and N. Chandra, Phys. Rev. A 4, 796 (1971).

[8] G. S. Agarwal, Lett. Nuovo Cimento 1, 53 (1971).

[9] J. Lehner, U. Leonhardt, and H. Paul, Phys. Rev. A 53, 2727 (1996).

[10] J. Söderholm, G. Björk, and A. Trifonov, Opt. Spectrosc. 91, 532 (2014).

[11] C. R. Müller, B. Stoklasa, C. Peuntinger, C. Gabriel, J. Řeháček, Z. Hradil, A. B. Klimov, G. Leuchs, C. Marquardt, and L. L. Sánchez-Soto, New J. Phys. 14, 085002 (2012).

[12] L. L. Sánchez-Soto, A. B. Klimov, P. de la Hoz, and G. Leuchs, J. Phys. B 46, 104011 (2013).

[13] P. de la Hoz, A. B. Klimov, G. Björk, Y. H. Kim, C. Müller, C. Marquardt, G. Leuchs, and L. L. Sánchez-Soto, Phys. Rev. A 88, 063803 (2013).

[14] K. Blum, Density Matrix Theory and Applications (Plenum, New York, 1981).

[15] D. N. Klyshko, Phys. Lett. A 163, 349 (1992).

[16] D. M. Klyshko, JETP 84, 1065 (1997).

[17] A. Luis and L. L. Sánchez-Soto, Prog. Opt. 41, 421 (2000).

[18] J. Schwinger, in Quantum Theory of Angular Momentum, edited by L. C. Biedenharn and H. Dam (Academic, New York, 1965).

[19] M. G. Raymer, D. F. McAlister, and A. Funk, in Quantum Communication, Computing, and Measurement 2, edited by P. Kumar (Plenum, New York, 2000).

[20] V. P. Karassiov and A. V. Masalov, JETP 99, 51 (2004).
[21] D. A. Varshalovich, A. N. Moskalev, and V. K. Khersonskii, Quantum Theory of Angular Momentum (World Scientific, Singapore, 1988).

[22] E. T. Jaynes, Probability Theory: The Logic of Science (Cambridge University Press, Cambridge, 2003).

[23] F. T. Arecchi, E. Courtens, R. Gilmore, and H. Thomas, Phys. Rev. A 6, 2211 (1972).

[24] A. Perelomov, Generalized Coherent States and their Applications (Springer, Berlin, 1986).

[25] C. Marquardt, J. Heersink, R. Dong, M. V. Chekhova, A. B. Klimov, L. L. Sánchez-Soto, U. L. Andersen, and G. Leuchs, Phys. Rev. Lett. 99, 220401 (2007).

[26] G. K. Gupta, A. Kumar, and R. S. Singh, Opt. Commun. 284, 4951 (2011).

[27] J. Zimba, EJTP 3, 143 (2006).

[28] O. Giraud, P. Braun, and D. Braun, New J. Phys. 12, 063005 (2010).

[29] D. Baguette, T. Bastin, and J. Martin, Phys. Rev. A 90, 032314 (2014).

[30] G. Björk, J. Söderholm, L. L. Sánchez-Soto, A. B. Klimov, I. Ghiu, P. Marian, and T. A. Marian, Opt. Commun. 283, 4440 (2010).

[31] A. Peres, Quantum Theory: Concepts and Methods (Kluwer, New York, 2002).

[32] P. Usachev, J. Söderholm, G. Björk, and A. Trifonov, Opt. Commun. 193, 161 (2001).

[33] A. Sehat, J. Söderholm, G. Björk, P. Espinoza, A. B. Klimov, and L. L. Sánchez-Soto, Phys. Rev. A 71, 033818 (2005).

[34] J. Crann, R. Pereira, and D. W. Kribs, J. Phys. A 43, 255307 (2010).

[35] E. Bannai and M. Tagami, J. Phys. A 44, 342002 (2011).

[36] R. L. Stratonovich, Zh. Eksp. Teor. Fiz. 31, 1012 (1956) [Sov. Phys. JETP 4, 891 (1957)].

[37] F. A. Berezin, Commun. Math. Phys. 40, 153 (1975). 